\title{
Virtual Versus In-Personal Teaching of Infection Control Essentials: A Quasi-Experimental Nonequivalent Groups Design
}

\author{
Fatimah Alshamrani, Dalia Aljrary, and Sharafaldeen Bin Nafisah
}

\begin{abstract}
Background In setting of the current COVID-19 pandemic, it is crucial to endorse infection control competencies. However, whether virtual training is equivalent to in-person teaching to develop such competencies requires further elucidation.

Aim We aim to explore the effect of a brief, three-to-fiveminute training session on infection control competencies in the major area of emergency department infection control, using virtual versus in-person training.

Methods Two hospitals were chosen, and the study design was a quasi-experimental multi-centre nonequivalent groups design.

Result The learning score increased from 39.06\%, SD=17.18 (95\% CI 32.39-45.72) to 52.48\%, SD=26.48 (95\% CI 44.01-60.95) in the virtual training group, and from $47.86 \%, \mathrm{SD}=22.51(95 \%$ CI $41.47-54.26)$ to $\mathbf{7 9 . 6 5} \%$, SD=21.45 (95\% CI 70.14-89.16) after the in-person teaching. The mean difference between the two groups revealed a higher learning score using in-person teaching: $27.16 \% ; t(60)=-4.12, p=0.0001$.
\end{abstract}

Conclusion Infection control competencies are better acquired via in-person teaching than by virtual education.

Index Terms-Virtual teaching, infection control, COVID-19

\section{INTRODUCTION}

The practice of universal precautions for isolation has long been a standard part of daily routine in areas with high exposure rates, such as the emergency department. In the setting of the current pandemic, compliance with universal isolation precautions is necessary for all healthcare workers, for their own safety and that of their patients and colleagues. Knowledge of certain infection control measures is imperative; this includes knowledge of aerosol-generating procedures and the risk of exposure during airway management, correct donning (putting on) and doffing (removing) of personal protective equipment (PPE), and effective handwashing. Acquiring such knowledge requires teaching and guidance.

Virtual training is considered an effective tool for teaching medical students [1]. The flexibility and safety of this method were advocated in another study [2], especially when social distancing has become the norm; however, whether virtual teaching is comparable to, or more effective than, in-person teaching requires further elucidation.

A previous analysis noted that virtual training is more effective for teaching hand hygiene and general information

Fatimah Alshamrani is with Emergency Department, King Fahd Medical City, Saudi Arabia., e-mail: Fatimahmed433@gmail.com

Dalia Aljrary is with Emergency Department, King Saud Medical City, Saudi Arabia., e-mail: Aljrarydalia@gmail.com

Sharafaldeen Bin Nafisah is with Emergency Department, King Fahd Medical City, Saudi Arabia., e-mail: Dr.Sharafaldeen@yahoo.com about the chain of transmission [3]. Virtual training also enhanced the competency score when used as a refresher course [4], proving feasible and effective [5]. Here, we explore the effect of a brief, three-to-five-minute training session on infection control competency in the major area of emergency department infection control, and compare the competency scores of virtual versus in-person training.

\section{METHODS}

We used a multi-centre, quasi-experimental nonequivalent groups design. The emergency departments of two hospitals were selected randomly: a tertiary and a secondary hospital, both in Riyadh City, Saudi Arabia. Only emergency residents were invited to participate. Each participant received a preteaching survey; thereafter, teaching was conducted using either a virtual learning method (in hospital A) or in-person teaching (in hospital B).

The virtual training, led by one of the authors, included a two-minute video demonstration of donning and doffing and of how to perform effective hand hygiene, in addition to a two-minute presentation. These were adapted from CDC educational videos, and only the practical part of the videos was displayed [6], [7].

The in-person teaching involved a quick, three-to-fiveminute discussion about the sequence of donning and doffing, with a physical demonstration at the end of the discussion.

Knowledge of infection control was conveyed in both methods, including donning and doffing, hand hygiene, aerosolgenerating procedures (intubation and extubation, airway suctioning, manual ventilation, non-invasive positive pressure ventilation, nebuliser or high-flow oxygen therapy, and cardiopulmonary resuscitation) [8]. Methods for minimising aerosols during exposure were also discussed. We delayed the post-intervention survey to avoid availability bias.

\section{RESULTS}

The response rate was $75.9 \%$ in hospital $\mathrm{A}$ and $80 \%$ in hospital B. 32.5\% completed the survey a few days after the virtual discussion, while $31 \%$ completed it a few days after the in-person discussion. The mean baseline knowledge score for residents in the virtual training group was $39.06 \%$, $\mathrm{SD}=17.18$ (95\% CI 32.39-45.72), whereas for the in-person training group it was $47.86 \%, \mathrm{SD}=22.51(95 \%$ CI 41.47 54.26). The difference in baseline knowledge between the two groups was not statistically significant; $\mathrm{t}(76)=-1.79, \mathrm{p}=0.077$. 
After the training, the score increased to $52.48 \%, \mathrm{SD}=26.48$ (95\% CI 44.01-60.95) in the virtual training group, and to $79.65 \%, \mathrm{SD}=21.45$ (95\% CI 70.14-89.16) in the in-person group. The mean difference between the two groups revealed a higher learning score using in-person teaching: $27.16 \%$; $\mathrm{t}(60)=-4.12, \mathrm{p}=0.0001$. Figure 1. illustrates the difference in knowledge score between the two groups before and after training.

\section{DISCUSSION}

Virtual training has received considerable attention during the current pandemic due to its ease, feasibility and practicality. However, our analysis revealed that in-person training for infection control measures remains superior to virtual training. The learning score in our analysis increased significantly more in the in-person training group than in the virtual group. We can infer that a physical demonstration of donning and doffing results in greater knowledge gain than does a video demonstration. We may also infer that, despite the ongoing pandemic and the strict infection control measures applied in various hospitals, continuous in-person training should remain the standard.

Nonetheless, virtual training should not be disregarded. Such methods were found to be effective in several analyses and under various conditions [9], [10].

Our analysis ensured a comparable duration of teaching for both methods; even so, it revealed the superiority of inperson teaching. It could be that virtual teaching requires more time than in-person education, or perhaps combining the two methods might provide more significant knowledge gain, as occurred in one study [11]. Thus, another study might be warranted to evaluate virtual training of a longer duration.

In this analysis, we used infection control measures relating to emergency department procedures, including intubation, non-invasive airway ventilation, and the use of nebulisation. These are standard procedures, and emergency residents should be competent to conduct them, and to teach infection control measures to other specialities in their respective departments. We believe empowering the trainees to teach infection control measures in-person will increase their competency and that of their colleagues.

The generalisability of our findings stems from the acceptable follow-up rate, the comparable baseline knowledge between the two hospitals, and the control for availability bias. We urge other departments to measure the infection control competencies of their trainees using our survey, or to adapt it to fit the needs of their departments.

\section{CONCLUSION}

Continuous training on infection control measures is crucial for the containment of the COVID-19 pandemic. Our findings indicate that in-person infection control training is superior to virtual training among emergency trainees. Further studies are advocated to investigate knowledge retention after several periods, to facilitate planning of regular infection control training programmes.

\section{REFERENCES}

[1] Wilcha RJ. Effectiveness of Virtual Medical Teaching During the COVID-19 Crisis: Systematic Review. JMIR Med Educ. 2020 Nov 18;6(2):e20963. doi: 10.2196/20963. PMID: 33106227; PMCID: PMC7682786.

[2] Dost S, Hossain A, Shehab M, Abdelwahed A, Al-Nusair L. Perceptions of medical students towards online teaching during the COVID-19 pandemic: a national cross-sectional survey of 2721 UK medical students. BMJ Open. 2020 Nov 5;10(11):e042378. doi: 10.1136/BMJ open-2020-042378. PMID: 33154063; PMCID: PMC7646323.

[3] Atack L, Luke R. Impact of an online course on infection control and prevention competencies. J Adv Nurs. 2008 Jul;63(2):175-80. doi: 10.1111/j.1365-2648.2008.04660.x. PMID: 18638159.

[4] Atack L, Luke R. Improving infection control competency through an online learning course. Nurs Times. 2009 Feb 3-9;105(4):30-2. PMID: 19263775.

[5] Sharma R, Mohanty A, Singh V, S VA, Gupta PK, Jelly $\mathrm{P}$, Gupta P, Rao S. Effectiveness of Video-Based Online Training for Health Care Workers to Prevent COVID-19 Infection: An Experience at a Tertiary Care Level Institute, Uttarakhand, India. Cureus. 2021 May 1;13(5):e14785. doi: 10.7759/cureus.14785. PMID: 34094747; PMCID: PMC8169096.

[6] Demonstration of donning (putting on) personal protective equipment (PPE) [Internet]. [cited 2021 Jun 16]. Available

from:

https://www.youtube.com/watch?v=H4jQUBAlBrI

[7] Demonstration of doffing (taking off) personal protective equipment (PPE) [Internet]. [cited 2021 Jun 16]. Available from: https://www.youtube.com/watch? $\mathrm{v}=\mathrm{PQxOc} 13 \mathrm{DxvQ}$

[8] Jackson T, Deibert D, Wyatt G, Durand-Moreau Q, Adisesh A, Khunti K, Khunti S, Smith S, Chan XHS, Ross L, Roberts N, Toomey E, Greenhalgh T, Arora I, Black SM, Drake J, Syam N, Temple R, Straube S. Classification of aerosol-generating procedures: a rapid systematic review. BMJ Open Respir Res. 2020 Oct;7(1):e000730. doi: 10.1136/bmjresp-2020-000730. PMID: 33040021; PMCID: PMC7549490.

[9] Labeau SO, Rello J, Dimopoulos G, Lipman J, Sarikaya A, Oztürk C, Vandijck DM, Vogelaers D, Vandewoude K, Blot SI; EVIDENCE Research Team. The Value of E-Learning for the Prevention of Healthcare-Associated Infections. Infect Control Hosp Epidemiol. 2016 Sep;37(9):1052-9. doi: 10.1017/ice.2016.107. Epub 2016 May 13. PMID: 27174463.

[10] Ortega J, Cometto MC, Zárate Grajales RA, Malvárez S, Cassiani S, Falconi C, Friedeberg D, Peragallo-Montano N. Distance learning and patient safety: Report and evaluation of an online patient safety course. Rev Panam Salud Publica. 2020 May 20;44:e33. doi: 10.26633/RPSP.2020.33. PMID: 32435264; PMCID: PMC7236860.

[11] Caniza MA, Homsi M, Gonzalez M, Moreira DC, Tang L, Adderson E, et al. Development, implementation, and outcomes of a global infectious disease training course. Journal of Medical Education and Curricular Development. $2021 \mathrm{Jan} ; 8: 238212052110152$. 


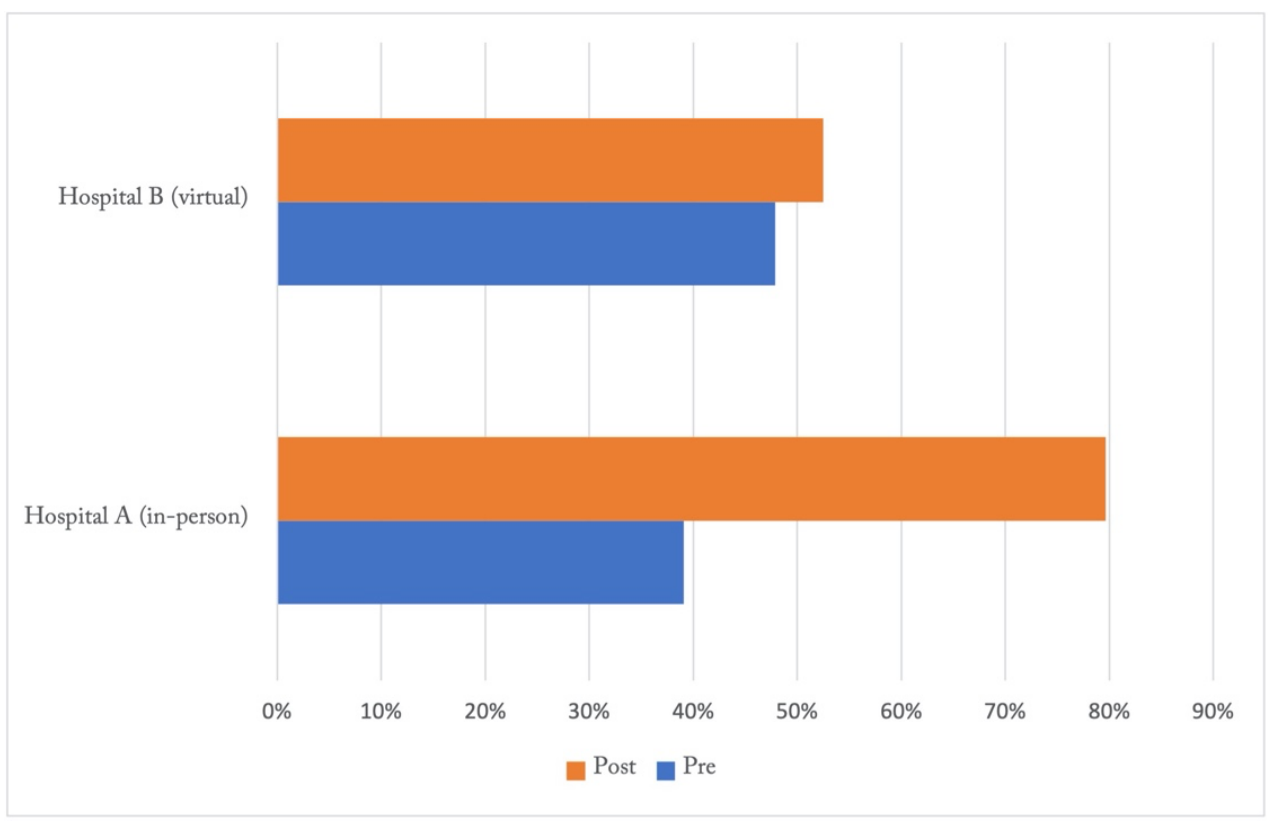

Figure 1. The difference inknowledge score before and after in-person and virtual training 